\title{
Descripción Anatómica de la Musculatura Intrínseca del Miembro Torácico de León (Panthera leo )
}

\author{
Anatomical Description of the Intrinsic Muscle of Thoracic Limb of Lion (Panthera leo )
}

Vargas J.F. ${ }^{1}$; Quintana O. ${ }^{1}$; Barraza C. ${ }^{1}$ \& Olivares R. ${ }^{2}$

VARGAS, J. F.; QUINTANA, O. ; BARRAZA, C. \& OLIVARES, R. Descripción anatómica de la musculatura intrínseca del miembro torácico de león (Panthera leo). Int. J. Morphol., 35(3):1154-1160, 2017.

RESUMEN: El presente estudio tuvo como objetivo el describir la musculatura intrínseca del miembro torácico del león africano (Panthera leo). Para tal efecto se utilizó un ejemplar hembra, donado por el Zoológico Metropolitano de Santiago-Chile, fijado y conservado. La disección se llevó a cabo, de proximal a distal, considerando las cuatro regiones topográficas de dicho miembro: Cintura Escapular, Brazo, Antebrazo y Mano. Se discuten y comparan los hallazgos anatómicos observados, con lo descrito para el gato doméstico y trabajos realizados por otros autores en Ocelote (Leopardus pardalis), Puma (Puma concolor) y en la propia especie en estudio. La finalidad del presente trabajo fue aportar al conocimiento de la anatomía de especies exóticas, con proyección médico quirúrgica.

PALABRAS CLAVES: Panthera leo, miembro torácico, músculos.

\section{INTRODUCCIÓN}

El león africano (Panthera leo), pertenece a la familia felidae del orden carnívora, una de las cuatro especies del género Panthera; es el segundo felino más grande después del tigre y puede alcanzar un peso entre 150-250 kg. Habita normalmente en África y Asia, pero en Chile se encuentra únicamente en estado de cautiverio.

Reportes de la Unión Internacional para la Conservación de la Naturaleza (UICN) del 2006, estimaban en 33.000 la cifra de leones salvajes en todo el continente africano, mientras que en el 2011 la cifra fue estimada en menos de 20.000 en toda la región (Riggio, 2011). La drástica disminución de estos animales, nos obliga a generar conocimiento sobre la biología del león africano y el aspecto anatómico no escapa a esta situación, ya que en particular el estudio de la musculatura de la familia de los felinos, esta pobremente documentada (Barone, 1967; Concha et al., 2004; Julik et al., 2012), y en los zoológicos frecuentemente se realizan diferentes procedimientos médicos, cada vez más complejos, siendo común la cirugía traumatológica a nivel de los miembros torácicos y pélvicos (Mudrovici, 2006).

Es por lo anterior que el objetivo del presente trabajo fue describir anatómicamente, los músculos intrínsecos del miembro torácico de un ejemplar de león africano (Panthera leo). Correspondiendo éstos, a los músculos que se originan y se insertan en los huesos que conforman dicho miembro (König \& Liebich, 2011). Para luego discutir lo visualizado, con lo reportado por Barone para el León, Concha et al., para el Puma (Puma concolor), Julik et al., para el Ocelote (Leopardus pardalis) y para gato y carnívoros doméstico (Getty, 1982; St. Claire 1982; Shaller, 1996; Done et al., 2010; Dyce et al., 2012; König \& Liebich; Evans \& de Lahunta, 2013).

\section{MATERIAL Y MÉTODO}

Para el presente estudio, se utilizó un ejemplar de león africano hembra adulto de $130 \mathrm{~kg}$, donado por el Zoológico Metropolitano de Santiago. El cadáver se fijó y conservó en la Unidad de Anatomía Veterinaria de la Universidad Santo Tomás, sede Santiago. Posteriormente, además, se realizó repleción intravascular con látex mezclado con tinta china roja para arterias y azul para venas (Medina et al., 2014).

Se procedió a la disección de proximal a distal, de la musculatura intrínseca de ambos miembros torácicos, con- 
siderando las cuatro regiones topográficas: Cintura Escapular, Brazo, Antebrazo y Mano. Respetando la vascularización e inervación regional.

\section{RESULTADOS Y DISCUSIÓN}

\section{Músculos de la región de la cintura escapular (Fig. 1).}

M. deltoides (Fig. 1A): La porción escapular, abarca los dos tercios distales de la espina escapular y parte del proceso suprahamata. La porción acromial más desarrollada, se origina en el acromion y proceso suprahamata. En su margen caudal hay una unión tendinosa con la cabeza lateral del tríceps braquial. Se inserta en la tuberosidad deltoidea. Barone, indica que el origen de la porción escapular incluye la totalidad de la espina escapular. Julik et al. lo limita sólo al proceso suprahamata y superficie del músculo infraespinoso. Lo observado coincide con Concha et al., y König \& Liebich.

M. supraespinoso (Fig. 1A): Se origina en la fosa supraespinosa y en la espina escapular. Se inserta en el tubérculo mayor del húmero. Barone, describe una fascia que pa- rece dividir en dos, el tercio distal del músculo. Julik et al. indican que es parcialmente divisible en superficial y profundo, lo que fue evidenciado en forma tenue es este estudio.

M. infraespinoso (Fig. 1A): Se origina en la fosa infraespinosa y en la espina escapular. Se inserta en el tubérculo mayor. Lo anterior coincide con Barone, Julik et al., Concha et al., y König \& Liebich.

M. redondo menor: Se origina en el tercio distal del margen caudal de la escápula. Su inserción es lateral al cuello del húmero, en el tubérculo menor. Esto es similar a lo descrito por Barone, König \& Liebich y Concha et al. Julik et al., indican que tiene inserción en el tubérculo mayor del húmero.

M. subescapular (Fig. 1B): Se origina en la fosa subescapular de la escápula. Sus haces, en número de cinco, se reúnen hacia distal en un fuerte tendón que se inserta en el tubérculo menor. Esto es similar a lo descrito por Barone, Schaller y Concha et al. Respecto al número de haces, Julik et al., describen seis en el miembro izquierdo y nueve en el derecho.

M. redondo mayor (Figs. 1A y B): Se origina en el tercio
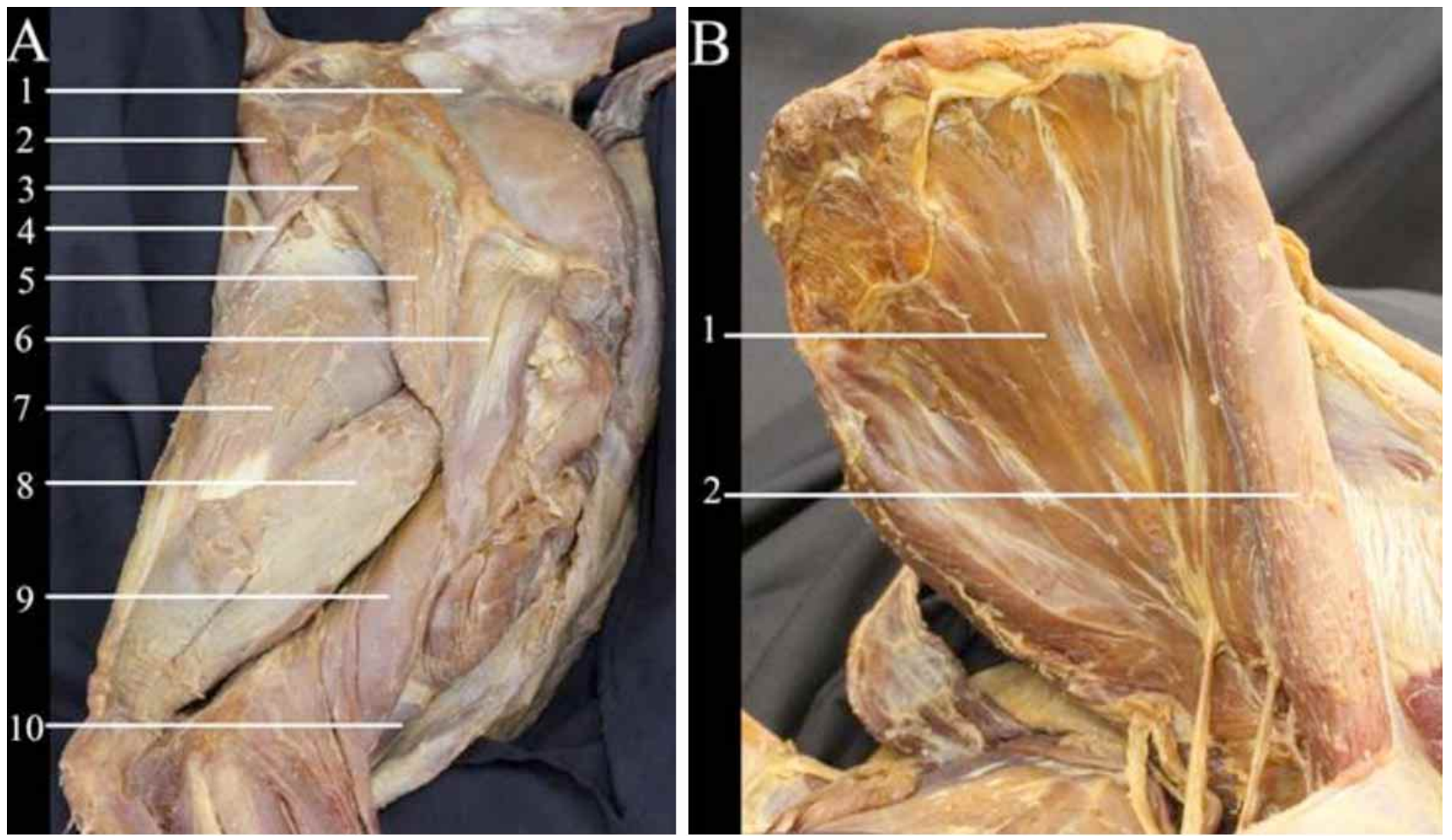

Fig. 1: Músculos de la región de la cintura escapular y el brazo, miembro torácico derecho A) Vista lateral 1) M. supraespinoso 2) M. redondo mayor 3) M. infraespinoso 4) M. tensor de la fascia antebraquial 5) M. deltoides, porción escapular 6) M. deltoides, porción acromial 7) Cabeza larga del tríceps braquial 8) Cabeza lateral del tríceps braquial 9) M. braquial 10) M. bíceps braquial B) Vista medial 1) M. subescapular 2) M. redondo mayor. 
proximal del margen caudal y el ángulo caudal de la escápula. Se inserta en la tuberosidad del redondo mayor del húmero. Barone indica un origen desde la mitad superior del margen caudal de la escápula. Julik et al., incluye la unión al músculo infraespinoso. En cuanto a la inserción existe coincidencia con Barone, Julik et al y Concha et al. Mientras que Schaller, indica que es en la cresta del tubérculo menor del húmero.

M. coracobraquial: Se origina en el proceso coracoides de la escápula y se inserta craneal al origen de la cabeza accesoria del tríceps braquial y distal al tubérculo menor. Barone y Concha et al., sitúan la inserción proximal y craneal a la del redondo mayor y dorsal ancho. König \& Liebich, indican que es distal a la tuberosidad del redondo mayor.

M. articular del húmero: Se origina en el cuello de la escápula. Lo anterior coincide con Barone, asegurando que la inserción tendría lugar en la cara caudal del húmero a poca distancia de la cabeza articular.

\section{Músculos de la región del brazo (Fig. 1).}

M. tensor de la fascia antebraquial (Fig. 1A): Se origina en la espina escapular, el músculo dorsal ancho y margen caudal de la escápula. Estas partes se unen para llegar al olécranon, caudal a la inserción del tríceps braquial, similar a lo descrito por Barone, Schaller y Julik et al. Concha et al., describen además un origen en el músculo redondo mayor.

M. tríceps braquial (Fig. 1A): Cabeza larga, se origina en la mitad distal del margen caudal de la escápula. Cabeza lateral, lateral al cuello humeral. Cabeza medial, en el tercio medio del húmero. Cabeza accesoria, en el cuello del húmero. Todas se unen al llegar al olécranon a través de un tendón muy fuerte. Esta descripción coincide con Barone. Julik et al., suman una quinta cabeza que denominan cabeza medial accesoria. Concha et al., describen sólo 3 cabezas. Schaller, sitúa el origen de la cabeza medial en la cresta del tubérculo menor.

M. Ancóneo: Se origina en el margen lateral de la fosa olecraneana y parte de la cresta supracondilar lateral. Se inserta en el olécranon en la superficie lateral. Esto coincide con Barone, Concha et al., y Schaller. Por otro lado, Barone, Julik et al., y Concha et al., coinciden con este trabajo, en que se puede evidenciar una cabeza lateral.

En una disposición similar a la del ancóneo, pero en la cara medial del codo, se encuentra un músculo delgado, cuyo origen se sitúa en el epicóndilo medial del húmero y en el margen medial del agujero supracondilar, para luego dirigirse hacía caudal e insertarse en el olécranon en su cara medial. Barone, describe algo similar, dándole el nombre de bajo ancóneo medial (Fig. 2B). Julik et al., hacen referencia a un músculo en situación similar pero más voluminoso y lo consideran como la quinta cabeza del tríceps (cabeza medial accesoria). St. Claire y Done et al., lo llaman músculo epitrocleoancóneo.

M. bíceps braquial (Fig. 1A): Se origina en el tubérculo supraglenoideo de la escápula, deslizándose por el surco intertubercular del húmero. Se inserta en la tuberosidad radial. Lo anterior concuerda con Barone, Julik et al., Concha et al. y Schaller.

M. braquial (Fig. 1A): Se origina desde el surco del músculo braquial, en la cara caudal del tercio proximal del húmero. Se inserta junto con el músculo cleidobraquial en el tercio proximal de la ulna. Esto último coincide con Barone y Julik et al. Concha et al., lo sitúan cerca del proceso coronoides lateral y König \& Liebich, del proceso coronoides medial.

\section{Músculos de la región del antebrazo (Figs. 2 y 3).}

M. braquiorradial (Fig. 2A): Se origina en el tercio medio del húmero, lateral al músculo braquial. Luego desciende superficialmente insertándose proximalmente al proceso estiloideo del radio. Esto es similar a lo descrito por Barone. Para Julik et al., el origen sería en el tercio medio del húmero en su cara caudal, para Concha et al., también en el tercio medio, pero en su cara lateral y según König \& Liebich, en la cresta supracondilar lateral. En cuanto a la inserción todos coinciden que es en la cara medial de la epífisis distal del radio.

M. extensor radial del carpo (Figs. 2A y 3A): Tiene dos cuerpos cuyo origen común se ubica en la cresta supracondilar lateral del húmero. M. extensor radial del carpo largo: se inserta proximal en el metacarpo II. M. extensor radial del carpo breve (corto): se inserta proximal en el metacarpo III, pero más distal con respecto al anterior. Lo anterior es muy similar a lo indicado por Barone y Julik et al. Concha et al., indican el origen en el epicóndilo lateral del húmero. König \& Liebich, señalan que se pueden dar cualquiera de las dos situaciones. Julik et al., señalan un tendón muy plano y delgado que une ambos tendones en la mitad del antebrazo, lo que también se observó en este estudio.

M. extensor digital común (Figs. 2A y 3A): Se origina en la cresta supracondilar lateral, distal al origen del M. extensor radial del carpo. El número de tendones descritos en este estudio (5), coincide sólo con Dyce et al. Mientras que Barone, Julik et al., Concha et al. y König \& Liebich, señalan 4 tendones. En cuanto a la inserción de éstos, Barone, 


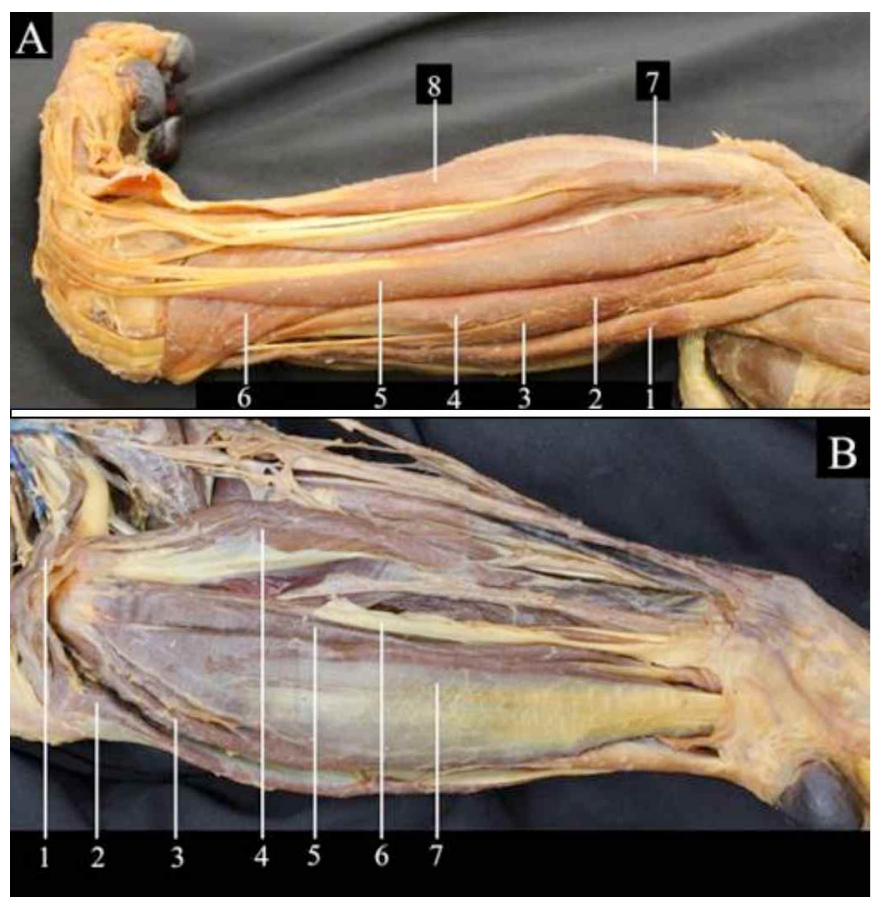

Fig. 2. Músculos de la región del antebrazo, miembro torácico derecho A) Vista craneolateral 1) M. Braquiorradial 2) M. Extensor radial del carpo 3) M. Extensor radial del carpo largo 4) M. Extensor radial del carpo breve 5) M. Extensor digital común 6) M. Abductor largo del dedo I 7) M. Extensor digital lateral 8) M. Extensor ulnar del carpo B) Vista medial 1) M. Ancóneo medial 2) Cabeza ulnar del flexor ulnar del carpo 3) Cabeza humeral del flexor ulnar del carpo 4) M. Pronador redondo 5) Cabeza humeral del flexor digital profundo 6) M. Flexor radial del carpo 7) M. Flexor digital superficial.

Julik et al., Concha et al., König \& Liebich y Dyce et al., señalan que se insertan en el proceso extensor de la falange distal de los dedos II, III, IV y V a diferencia de este estudio, que solo los logra seguir hasta el extremo distal de las falanges medias, lo que coincide con Dyce et al., y König \& Liebich.

M. extensor digital lateral (Figs. 2A y 3A): Se origina en el epicóndilo lateral del húmero. Se continúa por tres tendones que llegan al sesamoideo dorsal y la falange media de los dedos III, IV y V. A partir del tendón que va al dedo III, sale una rama tendinosa más delgada, a la altura del tercio proximal del metacarpo III, que va a la falange media del dedo II, previa unión al tendón del extensor digital I y II que llega al mismo dedo. Esto es similar a lo descrito por Barone y coincide con Concha et al., Julik et al., y König \& Liebich, que describen que el origen está en la cresta supracondilar lateral y además le describen tres cuerpos y Schaller agrega que hay una cuarta rama que se inserta en la falange media.

M. extensor ulnar del carpo (Figs. 2A y 3B): Se origina en el epicóndilo lateral del húmero. Termina en un tendón grueso y firme lateral al carpo y proximal en el metacarpo V. Esto coincide con Barone, Julik et al., Concha et al. y König \& Liebich. Julik et al., indican que en su inicio, esta fusionado con la cápsula articular del codo y al músculo ancóneo.

M. supinador: Tiene un origen fibroso en el epicóndilo lateral del húmero, cubierto por los músculos extensores digital lateral y común. Se inserta en el tercio medio del radio en su cara craneomedial. Barone, describe una inserción que abarca hasta el tercio distal del radio. Según Julik et al., se inicia en el ligamento anular y en el ligamento colateral lateral y se inserta en los dos tercios proximales del radio. Concha et al., coinciden con el presente estudio y Schaller, indica una inserción en el cuarto proximal del radio.

M. pronador redondo (Fig. 2B): Se origina en el epicóndilo medial del húmero. Su recorrido es oblicuo y con una inserción fibrosa y aplanada que llega al tercio medio del radio en su cara craneal. Barone, describe la inserción en el margen medial de la porción media del radio. Para Julik et al., ésta se ubica en los dos tercios distales del margen medial del radio, en el retináculo flexor y además en un sesamoideo ubicado en la base del primer metacarpiano. Según Concha et al., es en el tercio medio y distal del radio. Mientras que para König \& Liebich, la inserción se ubica en la cara craneomedial del radio.

M. abductor largo del dedo I (Fig. 2A): Su origen abarca prácticamente todo el margen lateral de la ulna y la mitad distal del radio. Luego pasa sobre los tendones del músculo extensor radial del carpo, insertándose en el metacarpo I. Para Barone, el origen es en el extremo proximal del margen lateral del radio y también la cara dorsal de la ulna. Para Julik et al., es en todo el margen lateral de la ulna, los dos tercios proximales del radio y la membrana interósea. Su inserción la describen en forma similar, agregando una extensión que llega a un pequeño sesamoideo metacarpal. Concha et al., y Schaller, indican que el origen es en el tercio medio de la ulna y en radio y ulna, respectivamente y su inserción también en la base del metacarpo I.

M. extensor digital I y II (Fig. 3A): Tiene un origen distal y lateral al olécranon y en los dos tercios proximales del margen lateral de la ulna. Su tendón llega a la falange proximal de los dedos I y II. Barone, indica que se origina en el margen lateral de la ulna, desde la parte superior del olécranon, cubriendo al músculo ancóneo. Sus tendones se insertan en la base del dedo I y extremo proximal del metacarpo II. Julik et al., indican su origen en la mi- 
tad proximal del margen lateral de la ulna. También señalan que hay dos cuerpos que emiten dos tendones, el que va al dedo II se une al tendón del músculo extensor digital lateral y la presencia de un tendón doble para el dedo I en el miembro izquierdo y uno solo para el miembro derecho del mismo espécimen. Concha et al., señalan que es un músculo pequeño que se origina en el tercio medio de la superficie lateral de la ulna y se inserta en los dedos I y II. König \& Liebich, indican la presencia de 3 ramas, donde dos de ellas llegan al dedo II.

M. flexor radial del carpo (Fig. 2B): Se origina en el epicóndilo medial del húmero. Termina insertándose en la base del metacarpo II. Esto coincide con lo descrito por Barone y Concha et al. König \& Liebich y Julik et al., agregan que la inserción es en el metacarpo II y III.

M. flexor digital superficial (Figs. 2B y 3B): Se origina en el epicóndilo medial del húmero. Emite un tendón que se divide en cinco ramas a nivel del carpo, para insertarse proximal en cada falange media de los dedos II al V y falange proximal del dedo I. Barone, indica que emite cuatro tendones a todos los dedos excepto al I y que la inserción es en la falange media. Julik et al., coincide con Barone, nombrando a este músculo como palmar largo. Concha et al., indican la inserción en los dedos II al V en las falanges proximales. Para König \& Liebich, este músculo emite cinco tendones, uno a cada dedo, hacia la falange media.

M. flexor ulnar del carpo (Figs. 2B y 3B): La Cabeza ulnar, se origina en el olécranon y la Cabeza humeral, en el epicóndilo medial del húmero. Se mantienen separadas hasta el tercio distal del antebrazo, donde se unen para originar un fuerte tendón que se inserta en el carpo accesorio. Además Barone indica, que en un tramo, se une a la cabeza humeral del flexor digital profundo.

M. flexor digital profundo (Figs. 2B y 3B): Cabeza humeral, se origina en el epicóndilo medial, y se compone a su vez de 3 cabezas. Cabeza radial, en el margen medio caudal del radio en los dos tercios proximales. Cabeza ulnar, en el olécranon ocupando los dos tercios proximales de la ulna por todo su margen caudal. Todas se unen para generar un tendón grueso, aplanado y muy fuerte que emite ramas a los procesos flexores de las falanges distales de todos los dedos. Barone, agrega que el origen de la cabeza humeral está muy asociado al origen del músculo flexor ulnar del carpo y además, especifica que la cabeza radial emite el tendón para el dedo I, si bien este trabajo confirma esto último, esto no es exclusivo, pues se evidencian fibras en otros sentidos. Julik et al., denominan a las cabezas humerales como humerales lateral, medial y profunda, todas originadas en el epicóndilo medial. Además, in- dican que la cabeza lateral recibe fibras musculares de la cabeza radial y ulnar. Esta última tiene tres orígenes: margen caudal de la ulna, cara caudal y caudomedial del radio y en la membrana interósea. La inserción es igual que lo señalado por este estudio, agregando que a diferencia de Barone, la cabeza humeral medial forma el tendón del dedo I. Concha et al., coinciden con el número de cabezas, dividiendo la humeral en cabezas 2, 3 y 4, nomenclatura que se adoptó en este estudio. Mientras König \& Liebich, describen sólo una cabeza humeral.

M. pronador cuadrado: Ocupa el espacio entre el radio y la ulna, cubierto por una gruesa membrana interósea. Barone, especifica que abarca los dos tercios distales de la cara palmar del radio y la ulna. Julik et al., coinciden en la ubicación, señalando que se origina distal en la ulna para insertarse distal en el radio. Para Concha et al., la situación es similar pero no comentan la presencia de una gruesa membrana interósea.

\section{Músculos de la mano (Fig. 3)}

M. interflexores (Fig. 3B): Se originan en la cara palmar del tendón flexor digital profundo, cabeza humeral, a la altura del tercio distal del radio. Se insertan, mediante tres tendones, a los dedos II, III y IV, junto con los tendones del flexor digital superficial. El tendón que va al dedo IV se fusiona con el tendón del flexor digital breve, que también llega al cuarto dedo, coincidiendo con Schaller. Barone, asocia este músculo a la cabeza ulnar del flexor digital profundo. Julik et al., precisan que posee tres vientres que se insertan juntos con los del flexor digital superficial llegando a los dedos II al IV.

M. flexor digital breve (corto) (Fig. 3B): Tiene dos cuerpos, uno emerge desde la superficie del tendón del flexor digital superficial, un poco antes de llegar al carpo y el otro tiene origen en el retináculo flexor, se dirige hacia distal aportando al mismo tendón con el que llegan al dedo IV. Este músculo emite un segundo tendón más superficial que llega al V dedo. Barone, agrega que los dos fuertes tendones penetran bajo el tendón del flexor digital superficial y que van a insertarse en los dedos IV y V, llamándolo palmar largo. Julik et al., indican que tiene tres vientres, dos superficiales y uno profundo. Los superficiales emiten un tendón al dedo $\mathrm{V}$, mientras que el profundo origina un tendón al dedo IV. Para Getty, se origina en la superficie profunda del flexor digital superficial y tiene una inserción en el ligamento transverso de la articulación metacarpofalángica de los dedos III, IV y V.

Mm. lumbricales (Fig. 3B): Son cuatro vientres que se originan a la altura del carpo desde la cara palmar del tendón 

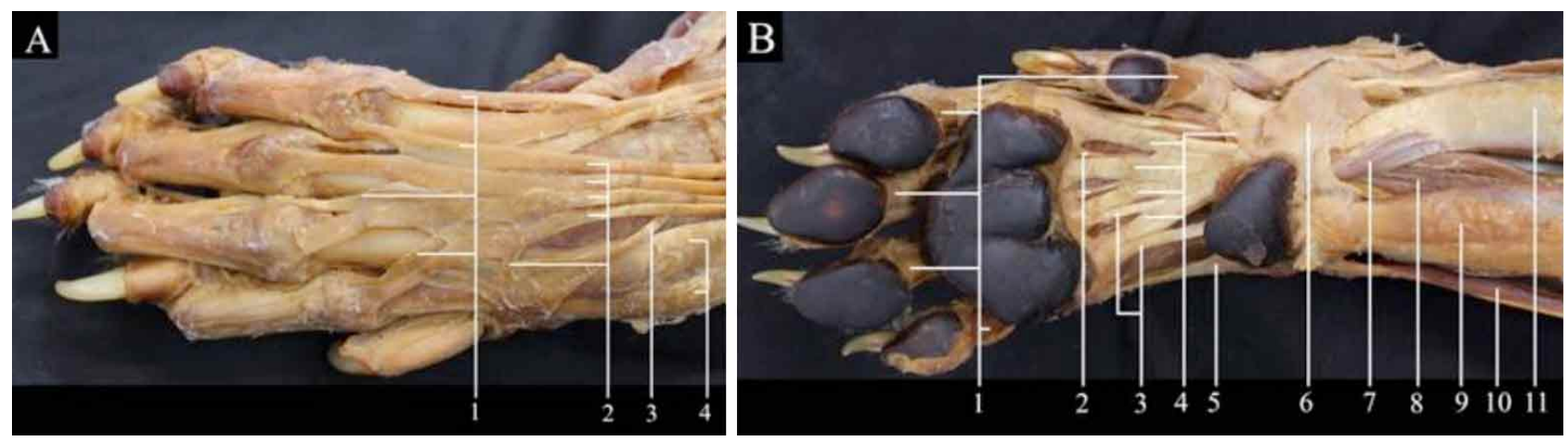

Fig. 3. Tendones de los músculos, mano derecha A) Vista dorsal 1) Tendones del músculo extensor digital lateral 2) Tendones del m. extensor digital común 3) Tendón del m. extensor digital I y II 4) Tendones del m. extensor radial del carpo B) Vista palmar 1) Tendones del $\mathrm{m}$. flexor digital profundo 2) Tendones de los mm. lumbricales 3) Tendones del $\mathrm{m}$. flexor digital breve (corto) 4) Tendones del $\mathrm{m}$. flexor digital superficial 5) Tendón del abductor digital V 6) M. flexor digital breve 8) Mm. interflexores. 9) Tendón del m. flexor ulnar del carpo 10) M. extensor ulnar del carpo 11) Tendón del flexor digital superficial.

del flexor digital profundo y se insertan en los tendones del flexor digital superficial. Barone, indica que éstos se ubican donde los flexores emiten sus ramas a los dedos. El más medial de ellos se ubica en el margen medial de los tendones del dedo II. Julik et al., señalan que hay 4 vientres distinguibles, con tendones a los dedos II al V y el más medial se ubica en el tendón del dedo I. Getty y Schaller, indican que tienen inserción en la superficie media proximal de la falange proximal de los dedos II al V.

Mm. interóseos: Estos cuatro músculos tienen origen en la base de los metacarpos II al V, coincidiendo con Barone. Un poco antes de llegar a su punto de inserción cada uno de éstos se divide en dos para insertarse finalmente a cada lado de las articulaciones metacarpofalángicas. Julik et al., los nombran como flexores breves profundos, haciendo una descripción individual de cada uno de ellos. Getty y Schaller, especifican que la inserción es en los sesamoideos proximales y en los tendones del extensor digital común.

Mm. abductor y flexor digital I breve: Se originan en el ligamento palmar del carpo, insertándose en la cara abaxial y axial, respectivamente y proximal de la falange proximal.

M. adductor digital I: Se origina en el ligamento palmar del carpo. Su inserción es en la cara axial de la falange proximal. La descripción de estos 3 últimos músculos, coincide con lo descrito por Barone y Schaller.

M. abductor digital V (Fig. 3B): Se origina en la cara palmar del carpo accesorio. Emite un tendón largo que se inserta en la cara abaxial de la falange proximal del dedo V. Barone, agrega en el origen al ligamento accesoriometacarpiano. Julik et al., incluyen al carpo accesorio, la base del IV y V metacarpiano y el tendón de inserción del músculo extensor ulnar del carpo.
M. flexor digital V: Se origina en la base del metacarpo V y se inserta en la falange proximal del dedo V. Barone, precisa que se ubica profundo y distal con respecto al abductor digital V. Getty sitúa su origen en el ligamento distal del carpo accesorio y la inserción en el sesamoideo lateral, además de la falange proximal del dedo $\mathrm{V}$.

M. adductor digital V: Se origina en la base del metacarpo V. Discurre paralelo al flexor digital V y su inserción es axial en la falange proximal. Barone, indica que el músculo es bífido con una inserción larga en el lado axial, en la parte más proximal de la falange proximal, al igual que lo observado en este estudio, y agrega una inserción más corta en el margen medial del metacarpo V. Julik et al., indican que posee dos vientres, lateral y medial que están unidos en su tercio proximal. Se originan desde la cara palmar del carpo III y se inserta el vientre lateral en el metacarpo $\mathrm{V}$ y el vientre medial en la base de la falange proximal del dedo V. Schaller, describe que el origen es en el retináculo flexor y la inserción en el lado axial de la falange proximal del dedo $\mathrm{V}$.

M. adductor digital II: Se origina en la base del metacarpo II. Su inserción es en la falange proximal del dedo II. Julik et al. y Schaller, señalan que se origina en el retináculo flexor cerca de la cara palmar del carpo III y se inserta en la base de la falange proximal del dedo II.

M. palmar breve: Está compuesto por fibras musculares que se originan en el retináculo flexor y se insertan en la almohadilla carpiana, coincidiendo con Barone. Julik et al., indican que está asociado a la almohadilla metacarpiana.

Finalmente como conclusión, podemos indicar que se identificó el origen, recorrido e inserción de los músculos que conforman la musculatura intrínseca del miembro torácico de león, donde se pudo apreciar similitudes y algu- 
nas diferencias entre lo descrito en este estudio y lo indicado por la bibliografía consultada para gato doméstico, la misma especie y otros felinos salvajes.

\section{AGRADECIMIENTOS}

Sr. Carlos Muñoz G. Técnico Laboratorio Unidad de Anatomía Veterinaria Sede Stgo. UST.

Dr. Ismael Concha A. College Veterinary Medicine, Lincoln Memorial University, Tennessee, USA.

VARGAS，J. F.; QUINTANA，O. ; BARRAZA，C.\& OLIVARES, R. Anatomical description of the intrinsic muscle of thoracic limb of lion (Panthera leo). Int. J. Morphol., 35(3):11541160, 2017.

SUMMARY: The objective of this study was to describe the intrinsic muscles of the thoracic limb of the African lion (Panthera leo). For this purpose a fixed and preserved female specimen donated by the Metropolitan Zoo in Santiago, Chile, was used. The dissection was carried out, from proximal to distal, considering the four topographical regions of that limb: Scapular girdle, arm, forearm and hand. Anatomical findings observed are discusses and compared to those described for the domestic cat and works by other authors in ocelot (Leopardus pardalis), puma (Puma concolor) and the species under study. The purpose of this work was to contribute to the knowledge of the anatomy of this exotic species, with medical and surgical projections.

KEY WORDS: Panthera leo; Thoracic limb; Muscles.
Panamericana, 2011

Medina, P. R.; Morales, M. P.; Concha, A. I. \& Borroni, G. C. Anatomical Description of Pelvic Limb Innervation of African Lion (Panthera leo). Int. J. Morphol., 32(3):889-94, 2014.

Mudrovici, D. E. Clínica y cirugía en félidos salvajes. Redvet Rev. Electron. Vet., 7(2):1-9, 2006. Disponible en: http://www.veterinaria.org/revistas/ redvet/n020206/020610.pdf

Riggio, J. S. The African Lion (Panthera leo leo): A Continent-Wide Species Distribution Study and Population Analysis. Master of Environmental Management Degree. Durham, Nicholas School of the Environment, Duke University, 2011. Disponible en: http://dukespace.lib.duke.edu/ dspace/bitstream/handle/10161/3714/Riggio_MP.pdf?sequence=1.

Shaller, O. Nomenclatura Anatómica Veterinaria Ilustrada. Zaragoza, Acribia, 1996.

Dirección para correspondencia:

Dr. Ricardo Olivares Pérez-Montt

Depto. Patología Animal

Facultad Ciencias Veterinarias y Pecuarias

Universidad de Chile

Av. Santa Rosa 11735 La Pintana

Santiago-Chile

E-mail: rolivare@uchile.cl

Recibido : 01-06-2017

Aceptado: 25-07-2017

\section{REFERENCIA BIBLIOGRAFÍCAS}

Barone, R. La myologie du lion (Panthera leo). Mammalia, 31(3):459514, 1967.

Concha, I.; Adaro, L.; Borroni, C. \& Altamarino, C. Anatomical considerations over the intrinsic musculature of thoracic limb of puma (Puma concolor). Int. J. Morphol., 22(2):121-5, 2004.

Done, S. H.; Goody, P. C.; Stickland, N. C. \& Evans, S. A. Atlas en color de anatomía veterinaria: El perro y el gato. $2^{\mathrm{a}}$ ed. Barcelona, Elsevier, 2010.

Dyce, K. M.; Sack, W. O. \& Wensing, C. J. G. Anatomía Veterinaria. $4^{\mathrm{a}}$ ed. Ciudad de México, El Manual Moderno, 2012.

Evans, H. E. \& de Lahunta, A. Miller's Anatomy of the Dog. $4^{\text {th }}$ ed. St. Louis, Elsevier Saunders, 2013.

Getty, R. Sisson y Grossman: Anatomía de los animales domésticos. Tomo II. 5a ed. Barcelona, Masson, 1982.

Julik, E.; Zack, S.; Adrian, B.; Maredia, S.; Parsa, A.; Poole, M.; Starbuck, A. \& Fisher, R. E. Functional anatomy of the forelimb muscles of the ocelot (Leopardus pardalis). J. Mammal. Evol., 19(4):277-304, 2012.

König, H. E \& Liebich, H. G. Anatomía de los animales domésticos. Texto y Atlas en Color. Tomo I: Aparato locomotor. $2^{\mathrm{a}}$ ed. Madrid, Médica 